\title{
Soybean Cyst Nematode Reduces Soybean Yield Without Causing Obvious Aboveground Symptoms
}

J. Wang and T. L. Niblack, Department of Plant Microbiology and Pathology, and J. A. Tremain and W. J. Wiebold, Department of Agronomy, University of Missouri, Columbia 65211; G. L. Tylka and C. C. Marett, Department of Plant Pathology, Iowa State University, Ames 50011; G. R. Noel, USDA-ARS, Department of Crop Sciences, University of Illinois, Urbana 61801; and O. Myers and M. E. Schmidt, Department of Plant, Soil, and General Agriculture, Southern Illinois University, Carbondale 62901

\begin{abstract}
Wang, J., Niblack, T. L., Tremain, J. A., Wiebold, W. J., Tylka, G. L., Marett, C. C., Noel, G. R., Myers, O., and Schmidt, M. E. 2003. Soybean cyst nematode reduces soybean yield without causing obvious aboveground symptoms. Plant Dis. 87:623-628.

Field experiments were conducted at locations in northern and southern Illinois, central Iowa, and central Missouri from 1997 to 1999 to investigate the effects of Heterodera glycines on soybean growth, development, and yield. A wide range of infestation levels was present at all locations. Two locally adapted cultivars, one resistant to $H$. glycines, were grown at each location. Cultivars were planted in alternating four-row strips with $76 \mathrm{~cm}$ between rows. For each cultivar, 201 -m-long single-row plots were sampled every 2 weeks starting 4 weeks after planting. Infection by $H$. glycines reduced plant height and leaf and stem weight on the resistant cultivars in the first 12 weeks after planting, and delayed pod and seed development 12 to 14 weeks after planting. Biomass accumulation was not reduced on the susceptible cultivars until 10 weeks after planting; reduction in pod and seed development occurred throughout the reproductive stages. Susceptible cultivars produced significantly lower yields than resistant cultivars, but the yield reductions were not accompanied by visually detectable symptoms.
\end{abstract}

The soybean cyst nematode, Heterodera glycines Ichinohe, is an economically important pathogen of soybean (Glycine max (L.) Merr.) in the north-central region of the United States, where most soybean production occurs (15). In Illinois, Iowa, and Missouri, the value of the estimated annual loss of soybean yield caused by $H$. glycines exceeds $\$ 300$ million (T. L. Niblack and G. L. Tylka, unpublished data). Currently, recommendations for management of $H$. glycines emphasize the use of resistant cultivars and incorporation of crop rotation with a nonhost in soybean production (10).

With its unique feeding site in soybean roots, $H$. glycines can impede soybean growth and development by interfering with root function. However, there is no consensus as to how and to what extent $H$. glycines affects plant growth and development. Early observations indicated that the

Corresponding author: T. L. Niblack

E-mail: tniblack@uiuc.edu

This research was supported by a grant from the Soybean Research and Development Council, and by the Agricultural Experiment Stations of Iowa State University, the University of Illinois, and the University of Missouri.

Accepted for publication 21 October 2002.

Publication no. D-2003-0324-01R

(C) 2003 The American Phytopathological Society effects of $H$. glycines on soybean growth were highly variable in the field, with no clear trend (3). In field microplots, H. glycines appeared to reduce plant height and biomass accumulation, but no significant reductions of these growth parameters were found in the field under different irrigation regimes (7). Increases in population densities of $H$. glycines were reported to reduce the number of pods in field microplots $(14,17)$. The "typical" aboveground symptoms of infection by $H$. glycines are stunting and chlorosis (1), yet significant yield loss in the absence of aboveground symptoms was observed in the north-central region (9). The difficulty of determining which aspect of soybean growth and development is most affected by $H$. glycines is an indication that interactions between $H$. glycines and environmental variables play an important role in determining soybean growth and development. Understanding soybean growth and development in the presence of $H$. glycines should help us design management plans to maximize soybean yield. A field project was conducted in Illinois, Iowa, and Missouri from 1997 to 1999 to investigate the effect of $H$. glycines on soybean growth, development, and yield by direct comparison of several biomass measurements and yield of both resistant and susceptible cultivars over the entire growing season. This project also generated data on soybean cyst nematode and plant biomass measure- ments as part of an effort to develop soybean growth models (5).

\section{MATERIALS AND METHODS}

Field sites naturally infested with $H$. glycines were chosen in northern and southern Illinois, central Iowa, and central Missouri. Conventional tillage was used at the Illinois and Iowa sites, whereas the Missouri site was no-till. Two locally adapted cultivars, one with resistance to $H$. glycines, were grown at each site (Table 1). Cultivars were planted in alternating fourrow strips with $76 \mathrm{~cm}$ between rows during the appropriate period for commercial soybean production at each site. Weed control at each site was accomplished with recommended herbicides, cultivation, or both.

A total of 500 1-m-long biomass sampling areas (plots) were marked at each location after planting, 250 in rows of the resistant cultivar and 250 in rows of the susceptible cultivar. Not every marked plot was sampled. A sufficient number of plots were marked so that the destructive sampling described in the following paragraph could take place throughout the growing season, with enough plots remaining to provide reasonable yield estimates.

Each 1-m-long plot contained at least 15 plants. Unsampled 1-m-long buffer areas separated each plot. The first sampling date was 4 weeks after planting and subsequent sampling dates followed at 2-week intervals until harvest. The number of sampling dates was different depending on field sites and growing seasons. At the Iowa site, the first sampling date in 1997 was 6 weeks after planting (WAP) for both cultivars, and samples for the susceptible cultivar at 20 WAP were missing (Table 2). There were eight and six sampling dates in 1998 and 1999, respectively (Table 2). At Missouri, there were nine sampling dates in 1997, and seven sampling dates completed at 16 WAP in 1998 and 1999 (Table 3 ). On each sampling date, the entire plot was rated for soybean growth stage (6), plant height (in centimeters), and number of plants. Before all plants in each sampling area were collected for bulk weight, three plants were chosen arbitrarily from each plot to measure leaf, stem, pod, and seed weights, and number of pods and seed were recorded after they were dried at 
$60^{\circ} \mathrm{C}$ for $72 \mathrm{~h}$. Plant weight of the entire plot, including three plants chosen for additional measurements, was recorded as bulk weight. Yields were based on plots harvested at physiological maturity for seed only. Seed collected from yield plots were sampled for seed composition analysis.

To ensure that each cultivar had comparable initial population densities (Pi) of $H$. glycines at each sampling date, the actual plots used for biomass measurement were not identified until the population density of $\mathrm{H}$. glycines in each plot was determined immediately after planting by the following method. Five soil cores $(19 \mathrm{~mm}$ in diameter by 15 to $20 \mathrm{~cm}$ ) were collected within a 7-cm range on either side of the seed row. Cysts were extracted from 100$\mathrm{cm}^{3}$ subsamples with a modified semiautomatic elutriator (2). Eggs were released from cysts with a motorized pestle (11) and counted under a dissecting microscope.

Based on the egg counts, plots of each cultivar were divided into groups. The frequency distribution of plots representing the overall range of $\mathrm{Pi}$ depended on year and location (Figs. 1 and 2). Plot assign- ments were based on five equal-sized groups for each year and location, as follows. The Pi were listed in order from low to high, with a cut-off at each value that represented one-fifth of the plots. The first group was labeled "A", the second group "B", and so on. Plots belonging to the same Pi group were randomly assigned a biomass sampling identification label from 1 to 10 , representing the sampling date. For each biomass sampling date, four plots of the same Pi group were randomly chosen so that 20 plots of each cultivar were sampled at each biomass sampling date (i.e., five infestation levels $\times$ four replications). The protocol guaranteed that plots chosen at each biomass sampling date had a similar Pi range of $H$. glycines from high to low; thus, plant biomass measurements across sampling dates were compared against a similar range of Pi. Actual values of $\mathrm{Pi}$, not $\mathrm{Pi}$ groups, were used for the regression analyses described below.

Means of biomass measurements (plant height; leaf, stem, pod, and seed weight; and number of seed) of two plots with the lowest $\mathrm{Pi}$ of $H$. glycines were used as base values to compute relative measurements.
Biomass measurements taken from the other remaining plots of each cultivar were divided by the corresponding means of biomass measurements obtained from the two plots with the lowest $\mathrm{Pi}$ to provide values of relative biomass measurements. Relative biomass measurements for individual plots at each site throughout the experiment were combined to evaluate the effect of $H$. glycines on soybean growth and development. Reductions in relative values reported here resulted from reductions in actual growth and development compared with those where Pi of $H$. glycines were the lowest. Therefore, relative values are indicators of soybean growth in the presence of $H$. glycines.

All Pi were transformed to $\log 10(x+1)$ values before analysis to reduce the correlation between means and variances. The effect of $H$. glycines on soybean growth and development over the entire growing season was evaluated by linear regression (SAS Institute, Cary, NC). Means of plant growth measurements and yields were separated with least-squares estimates of marginal means (LSMEANS) of the general linear models (GLM) procedure of SAS.

Table 1. Resistant (R) and susceptible (S) cultivars planted in northern and southern Illinois, central Iowa, and central Missouri in 1997 to 1999

\begin{tabular}{|c|c|c|c|c|c|c|}
\hline \multirow[b]{3}{*}{ Location } & \multicolumn{6}{|c|}{ Soybean cultivars } \\
\hline & \multicolumn{2}{|c|}{1997} & \multicolumn{2}{|c|}{1998} & \multicolumn{2}{|c|}{1999} \\
\hline & $\mathbf{R}$ & $\mathbf{S}$ & $\mathbf{R}$ & $\mathbf{S}$ & $\mathbf{R}$ & $\mathbf{S}$ \\
\hline Central Iowa & Jack & Kenwood 94 & Osage III & Mohegan II & Osage III & Mohegan III \\
\hline Northern Illinois & Linford & Williams 82 & Linford & Williams 82 & Linford & Williams 82 \\
\hline Southern Illinois & Fayette & Williams 82 & Fayette & Williams 82 & Fayette & Williams 82 \\
\hline Central Missouri & Pioneer 9362 & Pioneer 9381 & Pioneer 9362 & Pioneer 9381 & Pioneer 94B01 & Pioneer 9396 \\
\hline
\end{tabular}

Table 2. Means of plant height and biomass measurements of the resistant (R) and susceptible (S) cultivars at 4 to 22 weeks after planting during growing season at the Iowa site in 1997 to $1999^{z}$

\begin{tabular}{|c|c|c|c|c|c|c|c|c|c|c|c|c|}
\hline \multirow[b]{2}{*}{ Year } & \multirow[b]{2}{*}{ Biomass } & \multirow[b]{2}{*}{ Cultivar } & \multicolumn{10}{|c|}{ Weeks after planting } \\
\hline & & & 4 & 6 & 8 & 10 & 12 & 14 & 16 & 18 & 20 & 22 \\
\hline \multirow[t]{8}{*}{1997} & Height & $\mathrm{R}$ & - & 9.3 & 22.2 & 41.6 & $71.7 \mathrm{a}$ & $92.5 \mathrm{a}$ & $98.3 \mathrm{a}$ & $103.5 \mathrm{a}$ & $98.4 \mathrm{a}$ & $96.1 \mathrm{a}$ \\
\hline & $(\mathrm{cm})$ & $\mathrm{S}$ & - & 10.0 & 21.3 & 38.1 & $61.0 \mathrm{~b}$ & $76.1 \mathrm{~b}$ & $78.2 \mathrm{~b}$ & $72.05 \mathrm{~b}$ & $68.0 \mathrm{~b}$ & $63.5 \mathrm{~b}$ \\
\hline & Leaf wt. & $\mathrm{R}$ & - & 6.1 & 27.7 & $72.6 \mathrm{a}$ & $125.2 \mathrm{a}$ & $148.7 \mathrm{a}$ & $124.9 \mathrm{a}$ & $84.5 \mathrm{a}$ & 2.1 & 0.0 \\
\hline & $\left(\mathrm{g} / \mathrm{m}^{2}\right)$ & $\mathrm{S}$ & - & 6.5 & 24.4 & $59.8 \mathrm{~b}$ & $105.2 \mathrm{~b}$ & $104.1 \mathrm{~b}$ & $88.6 \mathrm{~b}$ & $35.8 \mathrm{~b}$ & - & 0.0 \\
\hline & Stem wt. & $\mathrm{R}$ & - & 2.0 & 15.2 & $55.9 \mathrm{a}$ & $139.2 \mathrm{a}$ & $242.7 \mathrm{a}$ & $210.1 \mathrm{a}$ & $217.5 \mathrm{a}$ & 142.4 & $116.7 \mathrm{a}$ \\
\hline & $\left(\mathrm{g} / \mathrm{m}^{2}\right)$ & $\mathrm{S}$ & - & 2.2 & 14.4 & $45.9 \mathrm{~b}$ & $110.2 \mathrm{~b}$ & $159.5 \mathrm{~b}$ & $132.0 \mathrm{~b}$ & $94.3 \mathrm{~b}$ & - & $61.5 \mathrm{~b}$ \\
\hline & Canopy wt. & $\mathrm{R}$ & - & 13.1 & 69.1 & 207.9 a & $437.3 \mathrm{a}$ & $699.4 \mathrm{a}$ & $830.1 \mathrm{a}$ & $1,037.7 \mathrm{a}$ & 850.5 & $708.2 \mathrm{a}$ \\
\hline & $\left(\mathrm{g} / \mathrm{m}^{2}\right)$ & $\mathrm{S}$ & - & 13.9 & 62.6 & $170.9 \mathrm{~b}$ & $356.1 \mathrm{~b}$ & $482.6 \mathrm{~b}$ & $542.3 \mathrm{~b}$ & $441.8 \mathrm{~b}$ & - & $331 \mathrm{~b}$ \\
\hline \multirow[t]{8}{*}{1998} & Height & $\mathrm{R}$ & $9.6 \mathrm{~b}$ & $17.6 \mathrm{~b}$ & 36.2 & 62.6 & 83.1 & 88.8 & 84.8 & $81.7 \mathrm{a}$ & - & - \\
\hline & $(\mathrm{cm})$ & $\mathrm{S}$ & $10.5 \mathrm{a}$ & $20.4 \mathrm{a}$ & 38.1 & 59.9 & 80.8 & 87.3 & 83.0 & $76.8 \mathrm{~b}$ & - & - \\
\hline & Leaf wt. & $\mathrm{R}$ & 9.2 & 15.9 & 33.0 & 75.2 & 114.6 & 120.9 & 85.4 & 0.0 & - & - \\
\hline & $\left(\mathrm{g} / \mathrm{m}^{2}\right)$ & $\mathrm{S}$ & 9.1 & 17.8 & 38.4 & 76.9 & 126.2 & 122.2 & 86.2 & 0.0 & - & - \\
\hline & Stem wt. & $\mathrm{R}$ & 3.8 & $9.5 \mathrm{~b}$ & 31.5 & 114.9 & 171.4 & 194.1 & 168.0 & 118.6 & - & - \\
\hline & $\left(\mathrm{g} / \mathrm{m}^{2}\right)$ & $\mathrm{S}$ & 4.3 & $12.3 \mathrm{a}$ & 36.5 & 107.2 & 188.5 & 195.9 & 177.4 & 119.7 & - & - \\
\hline & Canopy wt. & $\mathrm{R}$ & 11.1 & $22.5 \mathrm{~b}$ & 57.0 & 169.6 & 312.7 & $465.1 \mathrm{a}$ & $581.3 \mathrm{a}$ & $491.5 \mathrm{a}$ & - & - \\
\hline & $\left(\mathrm{g} / \mathrm{m}^{2}\right)$ & $\mathrm{S}$ & 11.3 & $26.1 \mathrm{a}$ & 64.7 & 158.9 & 304.4 & $408.4 \mathrm{~b}$ & $505.2 \mathrm{~b}$ & $442.4 \mathrm{~b}$ & - & - \\
\hline \multirow[t]{8}{*}{1999} & Height & $\mathrm{R}$ & 14.9 & $31.6 \mathrm{a}$ & 60.2 & 80.9 & 82.7 & 86.8 & - & - & - & - \\
\hline & $(\mathrm{cm})$ & $\mathrm{S}$ & 14.6 & $29.4 \mathrm{~b}$ & 57.9 & 81.6 & 85.4 & 84.8 & - & - & - & - \\
\hline & Leaf wt. & $\mathrm{R}$ & 10.8 & $46.0 \mathrm{a}$ & $95.7 \mathrm{a}$ & 163.0 & 142.1 & $44.5 \mathrm{~b}$ & - & - & - & - \\
\hline & $\left(\mathrm{g} / \mathrm{m}^{2}\right)$ & $\mathrm{S}$ & 9.5 & $38.1 \mathrm{~b}$ & $84.6 \mathrm{~b}$ & 145.7 & 146.1 & $62.4 \mathrm{a}$ & - & - & - & - \\
\hline & Stem wt. & $\mathrm{R}$ & 3.5 & 20.4 & 90.5 & $190.9 \mathrm{a}$ & 221.2 & 186.8 & - & - & - & - \\
\hline & $\left(\mathrm{g} / \mathrm{m}^{2}\right)$ & $\mathrm{S}$ & 3.2 & 16.8 & 81.0 & $159.7 \mathrm{~b}$ & 238.6 & 194.0 & - & - & - & - \\
\hline & Canopy wt. & $\mathrm{R}$ & 12.6 & $57.8 \mathrm{a}$ & $163.6 \mathrm{a}$ & $340.9 \mathrm{a}$ & 559.5 & 552.8 & - & - & - & - \\
\hline & $\left(\mathrm{g} / \mathrm{m}^{2}\right)$ & $\mathrm{S}$ & 11.3 & $47.8 \mathrm{~b}$ & $145.5 \mathrm{~b}$ & $287.1 \mathrm{~b}$ & 526.3 & 532.1 & - & - & - & - \\
\hline
\end{tabular}

\footnotetext{
${ }^{\mathrm{z}}$ Means separation was performed with the least-square means (LSMEANS) option of general linear model (GLM) procedure of SAS. Means followed by
} no letter within columns and the same year for each biomass measurement are not significantly different at $P=0.05$. 
Table 3. Means of plant height and biomass measurements of resistant (R) and susceptible (S) cultivars at 4 to 20 weeks after planting during growing season at the Missouri site in 1997 to $1999^{z}$

\begin{tabular}{|c|c|c|c|c|c|c|c|c|c|c|c|}
\hline \multirow[b]{2}{*}{ Year } & \multirow[b]{2}{*}{ Biomass } & \multirow[b]{2}{*}{ Variety } & \multicolumn{9}{|c|}{ Weeks after planting } \\
\hline & & & 4 & 6 & 8 & 10 & 12 & 14 & 16 & 18 & 20 \\
\hline \multirow[t]{8}{*}{1997} & Height & $\mathrm{R}$ & 11.2 & 20.3 & 46.7 & $70.0 \mathrm{a}$ & 75.9 & 77.4 & $71.6 \mathrm{a}$ & 69.1 & $70.5 \mathrm{a}$ \\
\hline & $(\mathrm{cm})$ & $\mathrm{S}$ & 11.4 & 19.7 & 46.4 & $62.5 \mathrm{~b}$ & 71.5 & 73.8 & $64.8 \mathrm{~b}$ & 65.0 & $64.3 \mathrm{~b}$ \\
\hline & Leaf wt. & $\mathrm{R}$ & 4.7 & 15.0 & 56.3 & $121.1 \mathrm{a}$ & 120.8 & 119.7 & $52.7 \mathrm{~b}$ & 0.0 & 0.0 \\
\hline & $\left(\mathrm{g} / \mathrm{m}^{2}\right)$ & $\mathrm{S}$ & 4.0 & 11.6 & 56.8 & $92.0 \mathrm{~b}$ & 109.0 & 127.9 & $85.2 \mathrm{a}$ & 2.8 & 0.0 \\
\hline & Stem wt. & $\mathrm{R}$ & 2.8 & $9.1 \mathrm{a}$ & 49.5 & $142.8 \mathrm{a}$ & $155.9 \mathrm{a}$ & 175.5 & 128.8 & 83.7 & $80.3 \mathrm{a}$ \\
\hline & $\left(\mathrm{g} / \mathrm{m}^{2}\right)$ & $\mathrm{S}$ & 2.3 & $6.5 \mathrm{~b}$ & 47.6 & $92.3 \mathrm{~b}$ & $121.8 \mathrm{~b}$ & 163.6 & 120.1 & 81.2 & $63.0 \mathrm{~b}$ \\
\hline & Canopy wt. & $\mathrm{R}$ & 6.6 & $21.0 \mathrm{a}$ & 92.7 & $238.8 \mathrm{a}$ & 313.6 & 472.0 & 473.1 & 381.7 & $375.1 \mathrm{a}$ \\
\hline & $\left(\mathrm{g} / \mathrm{m}^{2}\right)$ & $\mathrm{S}$ & 5.7 & $15.8 \mathrm{~b}$ & 90.2 & $165.8 \mathrm{~b}$ & 267.0 & 455.8 & 456.5 & 369.4 & $318.4 \mathrm{~b}$ \\
\hline \multirow[t]{8}{*}{1998} & Height & $\mathrm{R}$ & 13.0 & 34.1 & $61.8 \mathrm{~b}$ & 96.6 & $105.7 \mathrm{a}$ & $94.8 \mathrm{~b}$ & 95.3 & - & - \\
\hline & $(\mathrm{cm})$ & $\mathrm{S}$ & 15.1 & 30.8 & $71.5 \mathrm{a}$ & 90.4 & $99.4 \mathrm{~b}$ & $100.3 \mathrm{a}$ & 96.8 & - & - \\
\hline & Leaf wt. & $\mathrm{R}$ & 5.7 & 26.3 & $45.2 \mathrm{~b}$ & 77.2 & 92.8 & 77.0 & 0.0 & - & - \\
\hline & $\left(\mathrm{g} / \mathrm{m}^{2}\right)$ & $\mathrm{S}$ & 8.2 & 28.9 & $61.8 \mathrm{a}$ & 88.4 & 103.2 & 80.6 & 0.0 & - & - \\
\hline & Stem wt. & $\mathrm{R}$ & 3.4 & 19.8 & 76.4 & 208.7 & 296.6 & 267.1 & 166.8 & - & - \\
\hline & $\left(\mathrm{g} / \mathrm{m}^{2}\right)$ & $\mathrm{S}$ & 4.2 & 21.0 & 96.9 & 246.1 & 267.5 & 263.0 & 159.1 & - & - \\
\hline & Canopy wt. & $\mathrm{R}$ & 8.2 & 40.8 & $130.3 \mathrm{~b}$ & 324.1 & 566.2 & 707.4 & 568.7 & - & - \\
\hline & $\left(\mathrm{g} / \mathrm{m}^{2}\right)$ & $\mathrm{S}$ & 10.8 & 44.6 & $164.1 \mathrm{a}$ & 383.1 & 562.8 & 675.6 & 570.7 & - & - \\
\hline \multirow[t]{8}{*}{1999} & Height & $\mathrm{R}$ & $16.6 \mathrm{a}$ & 28.4 & $44.2 \mathrm{~b}$ & 72.8 & $83.1 \mathrm{a}$ & $86.3 \mathrm{a}$ & $82.1 \mathrm{a}$ & - & - \\
\hline & $(\mathrm{cm})$ & $\mathrm{S}$ & $14.8 \mathrm{~b}$ & 29.8 & $55.7 \mathrm{a}$ & 74.1 & $75.4 \mathrm{~b}$ & $77.8 \mathrm{~b}$ & $67.1 \mathrm{~b}$ & - & - \\
\hline & Leaf wt. & $\mathrm{R}$ & $10.8 \mathrm{a}$ & 30.8 & 77.3 & 132.6 & $154.2 \mathrm{a}$ & $148.5 \mathrm{a}$ & $62.2 \mathrm{a}$ & - & - \\
\hline & $\left(\mathrm{g} / \mathrm{m}^{2}\right)$ & $\mathrm{S}$ & $8.6 \mathrm{~b}$ & 28.6 & 73.9 & 122.5 & $127.3 \mathrm{~b}$ & $126.4 \mathrm{~b}$ & $33.1 \mathrm{~b}$ & - & - \\
\hline & Stem wt. & $\mathrm{R}$ & 4.9 & 19.8 & 68.5 & 149.1 & $192.9 \mathrm{a}$ & $216.2 \mathrm{a}$ & $173.9 \mathrm{a}$ & - & - \\
\hline & $\left(\mathrm{g} / \mathrm{m}^{2}\right)$ & $\mathrm{S}$ & 4.3 & 17.7 & 64.7 & 139.1 & $151.7 \mathrm{~b}$ & $164.0 \mathrm{~b}$ & $119.6 \mathrm{~b}$ & - & - \\
\hline & Canopy wt. & $\mathrm{R}$ & $13.6 \mathrm{a}$ & 44.9 & 128.8 & 261.4 & $371.2 \mathrm{a}$ & $519.3 \mathrm{a}$ & $503.9 \mathrm{a}$ & - & - \\
\hline & $\left(\mathrm{g} / \mathrm{m}^{2}\right)$ & $\mathrm{S}$ & $11.5 \mathrm{~b}$ & 40.0 & 122.6 & 237.9 & $331.0 \mathrm{~b}$ & $454.0 \mathrm{~b}$ & $391.6 \mathrm{~b}$ & - & - \\
\hline
\end{tabular}

${ }^{\mathrm{z}}$ Means separation was performed with the least-square means (LSMEANS) option of general linear model (GLM) procedure of SAS. Means followed by no letter within columns and the same year for each biomass measurement are not significantly different at $P=0.05$.

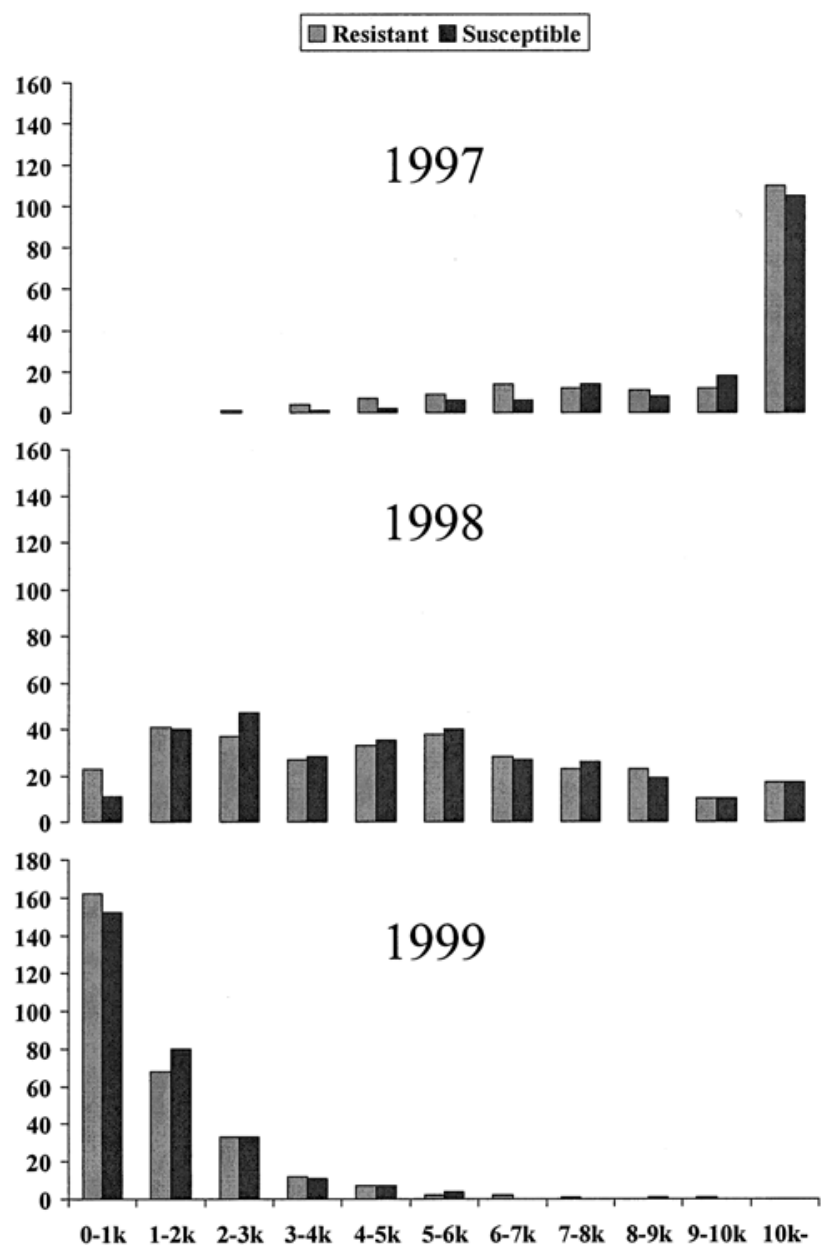

Fig. 1. Frequency distribution of initial population densities (eggs per $100 \mathrm{~cm}^{2}$ of soil) of Heterodera glycines at the beginning of each growing season in Iowa from 1997 to 1999.

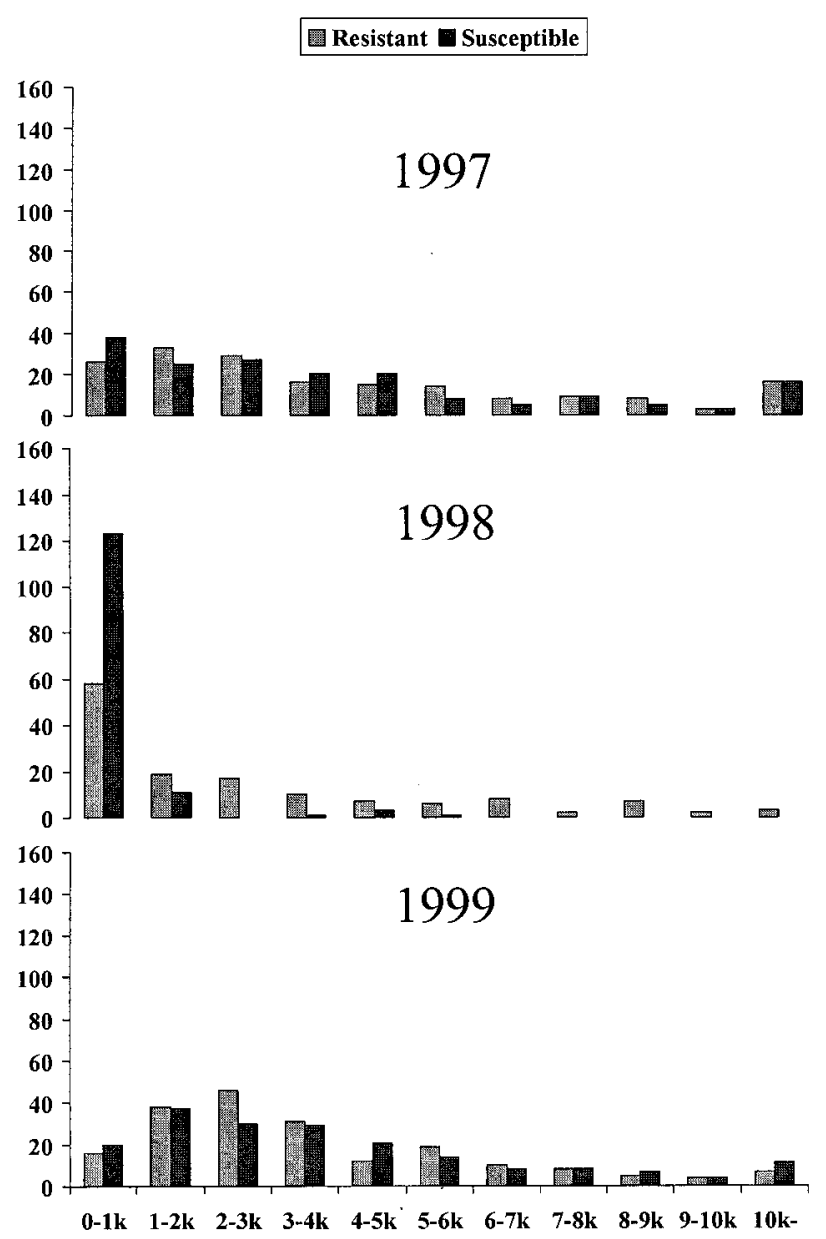

Fig. 2. Frequency distribution of initial population densities (eggs per $100 \mathrm{~cm}^{2}$ of soil) of Heterodera glycines at the beginning of each growing season in Missouri from 1997 to 1999. 


\section{RESULTS}

A range of Pi of $H$. glycines was present at each experimental site (Figs. 1 and 2). $\mathrm{Pi}$ of $H$. glycines in Iowa in 1997 were exceptionally high. Of the eight sampling dates for the susceptible cultivar and nine for the resistant cultivar completed in 1997 , only 15 of 340 plots had fewer than $5,000 \mathrm{eggs} / 100 \mathrm{~cm}^{3}$ of soil. No significant aggregation of $\mathrm{H}$. glycines was detected at any site (data not shown). A similar range of Pi was recorded for both cultivars except for Missouri in 1998, where the susceptible cultivar was planted inadvertently where the rows of the resistant cultivar had been in 1997.
At both the northern and southern Illinois sites, an unusually wet spring in 1997 and technical problems in 1998 resulted in datasets that were similar, but not directly comparable, to the datasets collected at the Missouri and Iowa sites. Separate analyses of the northern and southern Illinois datasets showed trends identical to those observed in the Missouri and Iowa sites. In order to reduce the complexity of this report, we include the analyses of the latter two only.

The effect of $H$. glycines on soybean growth and development was similar among locations (Tables 4 and 5). At the Iowa site, most of the reductions in plant height and leaf weight on the resistant cultivar occurred during the first 12 weeks after planting. The positive slopes of regression models after 12 weeks of planting suggest no further reductions in plant height, and leaf and stem weight as soybean progressed to maturity. As indicated by the negative slopes of the regression models, infestation delayed seed development between 12 and 14 weeks after planting; however, reductions were not observed after 14 weeks after planting. For the susceptible cultivar, reduction in plant height occurred 10 weeks after planting. Pod and seed development were reduced throughout the reproductive stages, par-

Table 4. Results of regression analysis of relative plant growth and development measurements of resistant (R) and susceptible (S) cultivars at 4 to 22 weeks after planting (WAP) during growing season against initial population density of Heterodera glycines at the Iowa site in 1997 to $1999^{z}$

\begin{tabular}{|c|c|c|c|c|c|c|c|c|c|c|c|c|c|}
\hline \multirow[b]{2}{*}{ Cultivar } & \multirow[b]{2}{*}{ WAP } & \multicolumn{2}{|c|}{ Plant height } & \multicolumn{2}{|c|}{ Leaf weight } & \multicolumn{2}{|c|}{ Stem weight } & \multicolumn{2}{|c|}{ Pod weight } & \multicolumn{2}{|c|}{ Seed weight } & \multicolumn{2}{|c|}{ No. of seeds } \\
\hline & & Slope $_{\mathrm{Pi}}$ & $R^{2}$ & Slope $_{\mathbf{P i}}$ & $R^{2}$ & Slope $_{\mathbf{P i}}$ & $R^{2}$ & Slope $_{\mathbf{P i}}$ & $R^{2}$ & Slope $_{\mathbf{P i}}$ & $R^{2}$ & Slope $_{\mathbf{P i}}$ & $R^{2}$ \\
\hline \multirow[t]{10}{*}{$\mathrm{R}$} & 4 & 0.18 & $0.34 *$ & 0.08 & 0.05 & 0.33 & $0.24 *$ & - & - & - & - & - & - \\
\hline & 6 & -0.02 & 0.01 & -0.17 & $0.20 *$ & 0.04 & 0.01 & - & - & - & - & - & - \\
\hline & 8 & -0.06 & $0.12 *$ & -0.13 & $0.16^{*}$ & -0.08 & 0.06 & - & - & - & - & - & - \\
\hline & 10 & -0.05 & $0.08^{*}$ & 0.07 & 0.02 & -0.12 & 0.05 & -0.45 & $0.25^{*}$ & - & - & - & - \\
\hline & 12 & -0.02 & 0.02 & -0.09 & $0.08^{*}$ & 0.01 & 0.00 & 0.12 & 0.05 & -0.72 & $0.61 *$ & -0.80 & $0.65^{*}$ \\
\hline & 14 & 0.07 & $0.14^{*}$ & 0.24 & 0.15 & 0.17 & $0.09 *$ & -0.01 & 0.00 & -0.61 & $0.53^{*}$ & -0.58 & $0.56^{*}$ \\
\hline & 16 & -0.08 & 0.09 & 0.00 & 0.00 & 0.01 & 0.00 & 0.09 & 0.03 & 0.10 & 0.02 & -0.05 & 0.01 \\
\hline & 18 & 0.07 & 0.05 & 1.17 & $0.49^{*}$ & 0.48 & $0.23^{*}$ & 0.33 & $0.18^{*}$ & 0.30 & $0.13^{*}$ & 0.33 & $0.15^{*}$ \\
\hline & 20 & 0.00 & 0.00 & 0.67 & 0.06 & 0.37 & 0.16 & 0.20 & 0.06 & 0.14 & 0.03 & 0.17 & 0.03 \\
\hline & 22 & 0.04 & 0.00 & - & - & 0.30 & 0.05 & 0.20 & 0.07 & 0.17 & 0.05 & -0.02 & 0.00 \\
\hline \multirow[t]{10}{*}{$S$} & 4 & 0.17 & $0.26^{*}$ & 0.18 & 0.11 & 0.18 & 0.08 & - & - & - & - & - & - \\
\hline & 6 & 0.09 & $0.19^{*}$ & 0.14 & $0.13^{*}$ & 0.43 & $0.27 *$ & - & - & - & - & - & - \\
\hline & 8 & 0.05 & 0.06 & 0.24 & $0.14^{*}$ & 0.30 & $0.15^{*}$ & - & - & - & - & - & \\
\hline & 10 & -0.07 & $0.09^{*}$ & 0.11 & 0.07 & -0.19 & $0.14 *$ & -0.60 & $0.40^{*}$ & - & - & - & - \\
\hline & 12 & -0.09 & $0.19^{*}$ & -0.05 & 0.02 & -0.11 & 0.07 & -0.14 & $0.11^{*}$ & -0.60 & $0.52 *$ & -0.67 & $0.55^{*}$ \\
\hline & 14 & -0.06 & $0.09 *$ & 0.10 & 0.06 & 0.01 & 0.00 & 0.02 & 0.00 & -0.45 & $0.46^{*}$ & -0.45 & $0.41 *$ \\
\hline & 16 & -0.22 & $0.21^{*}$ & -0.47 & $0.16^{*}$ & -0.25 & 0.09 & -0.18 & 0.04 & -0.21 & 0.04 & -0.13 & 0.02 \\
\hline & 18 & -0.18 & $0.25^{*}$ & 0.96 & $0.33^{*}$ & -0.29 & $0.13 *$ & -0.36 & 0.10 & -0.36 & 0.09 & -0.43 & $0.13 *$ \\
\hline & 20 & & & & & & & & & & & & \\
\hline & 22 & -0.27 & 0.06 & - & - & -0.79 & 0.06 & -0.96 & 0.12 & -1.01 & 0.13 & -1 & 0.13 \\
\hline
\end{tabular}

${ }^{\mathrm{z}}$ Regressions were performed on relative measurements and values of $\log 10(\mathrm{Pi}+1)$ for initial population density. Parameters (slope and $\left.R^{2}\right)$ presented in the table are for the linear models. $R^{2}$ followed by the asterisk indicates the linear model for the measurements at corresponding sampling dates are significant at $P=0.05$.

Table 5. Results of regression analysis of relative plant growth and development measurements of resistant (R) and susceptible (S) cultivars at 4 to 22 weeks after planting (WAP) during growing season on initial population density of Heterodera glycines at the Missouri site in 1997 to $1999^{z}$

\begin{tabular}{|c|c|c|c|c|c|c|c|c|c|c|c|c|c|}
\hline \multirow[b]{2}{*}{ Cultivar } & \multirow[b]{2}{*}{ WAP } & \multicolumn{2}{|c|}{ Plant height } & \multicolumn{2}{|c|}{ Leaf weight } & \multicolumn{2}{|c|}{ Stem weight } & \multicolumn{2}{|c|}{ Pod weight } & \multicolumn{2}{|c|}{ Seed weight } & \multicolumn{2}{|c|}{ No. of seeds } \\
\hline & & $\overline{\text { Slope }_{P i}}$ & $R^{2}$ & Slope $_{P i}$ & $R^{2}$ & Slope $_{\mathbf{P i}}$ & $R^{2}$ & Slope $_{P i}$ & $R^{2}$ & Slope $_{\mathbf{P i}}$ & $R^{2}$ & Slope $_{\mathbf{P i}}$ & $R^{2}$ \\
\hline \multirow[t]{9}{*}{$\mathrm{R}$} & 4 & -0.08 & 0.01 & -0.28 & 0.00 & 0.03 & 0.00 & - & - & - & - & - & - \\
\hline & 6 & -0.21 & $0.09 *$ & -0.36 & 0.04 & -0.34 & 0.02 & - & - & - & - & - & - \\
\hline & 8 & -0.04 & 0.01 & -0.05 & 0.01 & -0.05 & 0.01 & - & - & - & - & - & - \\
\hline & 10 & -0.05 & 0.05 & -0.05 & 0.01 & -0.16 & 0.05 & -0.31 & 0.07 & - & - & - & - \\
\hline & 12 & 0.03 & 0.02 & 0.12 & 0.05 & 0.04 & 0.00 & 0.07 & 0.01 & 0.03 & 0.00 & -0.1 & 0.01 \\
\hline & 14 & 0.00 & 0.00 & 0.04 & 0.01 & -0.01 & 0.00 & 0.06 & 0.01 & 0.20 & 0.06 & -0.02 & 0.00 \\
\hline & 16 & -0.06 & $0.12 *$ & 0.50 & 0.04 & 0.01 & 0.00 & 0.05 & 0.01 & 0.04 & 0.00 & 0.07 & 0.01 \\
\hline & 18 & 0.16 & 0.31 & - & - & -0.07 & 0.02 & 0.00 & 0.00 & 0.04 & 0.00 & -0.58 & 0.22 \\
\hline & 20 & 0.03 & 0.01 & - & - & -0.18 & 0.08 & 0.00 & 0.00 & -0.02 & 0.00 & 0.00 & 0.00 \\
\hline \multirow[t]{9}{*}{ S } & 4 & -0.31 & $0.39 *$ & 0.25 & $0.19^{*}$ & 0.31 & $0.22 *$ & - & - & - & - & - & - \\
\hline & 6 & 0.04 & 0.05 & 0.13 & $0.17 *$ & 0.13 & $0.17 *$ & - & - & - & - & - & - \\
\hline & 8 & 0.06 & 0.06 & 0.15 & 0.07 & 0.13 & 0.03 & - & - & - & - & - & - \\
\hline & 10 & -0.24 & $0.35^{*}$ & -0.34 & $0.31 *$ & -0.54 & $0.33^{*}$ & -0.24 & 0.04 & - & - & - & - \\
\hline & 12 & 0.01 & 0.01 & -0.02 & 0.00 & -0.03 & 0.01 & 0.15 & 0.08 & -0.39 & $0.4 *$ & -0.40 & $0.28^{*}$ \\
\hline & 14 & 0.00 & 0.00 & -0.10 & 0.05 & -0.06 & 0.02 & 0.00 & 0.00 & 0.10 & 0.07 & 0.00 & 0.00 \\
\hline & 16 & -0.03 & 0.03 & 0.87 & $0.45^{*}$ & -0.12 & $0.1 *$ & -0.39 & $0.47 *$ & -0.39 & $0.47 *$ & -0.20 & $0.25 *$ \\
\hline & 18 & -0.10 & 0.17 & - & - & -0.61 & $0.41 *$ & -0.47 & $0.52 *$ & -0.49 & $0.49 *$ & -0.52 & $0.29 *$ \\
\hline & 20 & -0.12 & 0.24 & - & - & -0.56 & 0.26 & -0.27 & 0.17 & -0.37 & 0.28 & -0.45 & $0.44 *$ \\
\hline
\end{tabular}

${ }^{\mathrm{z}}$ Regressions were performed on relative measurements and values of $\log 10(\mathrm{Pi}+1)$ for initial population density. Parameters $\left(\right.$ slope and $\left.R^{2}\right)$ presented in the table are for the linear models. $R^{2}$ followed by the asterisk indicates the linear model for the measurements at corresponding sampling dates are significant at $P=0.05$. 
ticularly between 12 and 14 weeks after planting (Table 4).

At the Missouri site, reductions in plant growth on the resistant cultivar were recorded in the first 10 weeks after planting, with significant reduction at 6 weeks after planting. Pod and seed development was not reduced. For the susceptible cultivar, reductions in plant growth occurred 10 weeks after planting. Stem weight was significantly reduced at 10,16 , and 18 weeks after planting. Significant reductions in pod and seed development occurred throughout reproductive development (Table 5). At both sites, slopes of linear models of soybean development measurements are steeper than those of soybean growth measurements except those for resistant cultivars in Missouri.

Plant height, leaf weight, stem weight, and canopy weight were used to measure soybean growth. The two cultivars followed similar growth patterns throughout the growing season (Tables 2 and 3). At the Iowa site, measurements for both cultivars were similar throughout biomass sampling except in 1997, when $\mathrm{Pi}$ of $H$. glycines were high (Table 2; Fig. 1). At the Missouri site, similar trends were observed (Table 3); however, the resistant cultivar had consistently higher growth measurements than the susceptible cultivar at later sampling dates in 1999.

Although differences in plant growth measurements were not consistent or significant, yields of resistant soybean cultivars were significantly greater than those of susceptible cultivars at most sites (Table 6). Seed composition, including protein, oil, fiber, saturated fatty acids, and total fatty acids between resistant and susceptible cultivars at all sites were not different (data not shown).

\section{DISCUSSION}

It is informative to have a wide range of $\mathrm{Pi}$ of $H$. glycines at all sampling dates when responses of both resistant and susceptible cultivars are evaluated throughout the soybean growing season. The experimental design we used resulted in a fairly similar range of $\mathrm{Pi}$ at all sampling dates with relatively easy field implementation. Our intensive field sampling indicated there is a wide range of $\mathrm{Pi}$ within relatively

Table 6. Means of soybean yield $(\mathrm{kg} / \mathrm{ha})$ for resistant $(\mathrm{R})$ and susceptible $(\mathrm{S})$ cultivars at four sites in 1997 to $1999^{z}$

\begin{tabular}{lccccc}
\hline Year & Cultivar & Central Iowa & Central Missouri & $\begin{array}{c}\text { Northern } \\
\text { Illinois }\end{array}$ & $\begin{array}{c}\text { Southern } \\
\text { Illinois }\end{array}$ \\
\hline 1997 & $\mathrm{R}$ & $4,171 \mathrm{a}$ & $2,361 \mathrm{a}$ & $3,161 \mathrm{a}$ & $2,316 \mathrm{a}$ \\
& $\mathrm{S}$ & $2,142 \mathrm{~b}$ & $2,082 \mathrm{~b}$ & $2,392 \mathrm{~b}$ & $2,354 \mathrm{a}$ \\
1998 & $\mathrm{R}$ & $3,360 \mathrm{a}$ & $3,304 \mathrm{a}$ & $3,056 \mathrm{a}$ & $2,918 \mathrm{a}$ \\
& $\mathrm{S}$ & $3,003 \mathrm{~b}$ & $3,337 \mathrm{a}$ & $2,432 \mathrm{~b}$ & $2,864 \mathrm{a}$ \\
1999 & $\mathrm{R}$ & $2,841 \mathrm{a}$ & $2,270 \mathrm{a}$ & $3,398 \mathrm{a}$ & $3,734 \mathrm{a}$ \\
& $\mathrm{S}$ & $2,357 \mathrm{~b}$ & $1,883 \mathrm{~b}$ & $3,140 \mathrm{a}$ & $3,288 \mathrm{~b}$ \\
\hline
\end{tabular}

${ }^{\mathrm{z}}$ Means separation was performed with the least-square means (LSMEANS) option of general linear models (GLM) procedure of SAS. Means followed by the same letter within columns and the same year and same site are not significantly different at $P=0.05$. small fields. To estimate field infestation with $H$. glycines, diagnostic field sampling must be carefully planned to avoid spots where $H$. glycines population densities may be at the lower end of the range. Underestimation of nematode pressure may lead to unpleasant economic consequences.

Soybean growth and development are determined by both genotype and environment. Even among closely related cultivars, significant differences in growth and development occur under similar growing conditions. To standardize our measurements, we used relative biomass measurements calculated from the corresponding measurements collected at the same time from the two plots that had the lowest Pi of $H$. glycines. In addition, relative values can be combined over years to evaluate the general effect of $H$. glycines, whereas the use of actual values may highlight heterogeneous variances that prevent combination over years. Relative values are an accepted means of assessing host responses to nematode infection $(12,13)$.

One of the more interesting results of this study was the observation that the growth of resistant cultivars was reduced during the first 12 weeks after planting. Resistance in soybean does not prevent penetration by second-stage juveniles of $H$. glycines (4). In fact, the root cells where initial syncytia are formed undergo degeneration and death within days after root penetration in resistant hosts (8). The hypersensitive-type responses may have created nutritional sinks at initial syncytia immediately after penetration in a resistant host, and growth reduction would be a reflection of this effect. With a limited number of fully developed syncytia in their root systems, resistant hosts eventually compensate for the reduction that occurred at the beginning, as indicated in the study. In contrast, the formation of syncytia in the roots of susceptible plants is much less detrimental to host tissue. No cell wall thickening and necrosis occurs during the early period of infection. The continual nematode feeding on susceptible hosts, however, eventually disrupts root function, deprives the host of nutrients, and results in reduction in soybean growth and development as indicated in our data.
One of the challenges in managing soybean cyst nematode is to convince producers to implement management practices before the damage becomes obvious. In our study, significant yield loss occurred in the absence of visually obvious symptoms such as stunting. Within the same range of Pi of $H$. glycines, slopes of linear models for measurements of plant height, leaf weight, and stem weight were flatter than those for pod weight, seed weight, and number of seed. This result suggests that reductions in components related to yield are not necessarily related to changes in plant growth. This is in agreement with Young (16), who observed that $H$. glycines can cause yield reduction in the absence of visible decline in growth. Little or no change in plant growth in the presence of $H$. glycines may belie the loss in yield. When resistant and susceptible cultivars were compared, significant differences in measurements, such as plant height, leaf, stem, and canopy weight at all sites, were not visible in the field unless the Pi of $H$. glycines were exceptionally low or high. The similar growth between the two cultivars can mislead growers to conclude that there is no gain to be had by switching to resistant cultivars. Our research indicated that resistant cultivars consistently produced higher yields than susceptible cultivars. The difference in yield between resistant and susceptible cultivars at the Iowa site in 1997 indicated that yield of resistant cultivars can be double that of susceptible cultivars if $\mathrm{Pi}$ are high. Carefully chosen resistant cultivars can benefit producers in H. glycines-infested fields even though no obvious growth difference is visible.

\section{ACKNOWLEDGMENTS}

We thank G. F. Krause, Department of Agronomy/Statistics, and M. R. Ellersieck, Experiment Station Statistics, University of Missouri, for their assistance and guidance in the statistical analyses of our data.

\section{LITERATURE CITED}

1. Agrios, G. N. 1997. Plant Pathology, 4th ed. Academic Press, New York.

2. Byrd, D. W., Jr., Barker, K. R., Ferris, H., Nusbaum, C. J., Griffin, W. E., Small, R. H., and Stone, C. A. 1976. Two semiautomatic elutriators for extracting nematodes and certain fungi from soil. J. Nematol. 8:206-212.

3. Dropkin, V. H., Baldwin, C. H., Gaither, T., and Nace, W. 1976. Growth of Heterodera glycines (soybean cyst nematode) in soybeans in the field. Plant Dis. Rep. 60:977-980.

4. Endo, B. Y., and Veech, J. A. 1970. Morphology and histochemistry of soybean roots infected with Heterodera glycines. Phytopathology 60:1493-1498.

5. Fallick, J. B., Batchelor, W. D., Tylka, G. L., Niblack, T. L., and Paz, J. O. 2002. Coupling soybean cyst nematode damage to CROPGRO-Soybean. Trans. Am. Soc. Agric. Eng. 45:433-441.

6. Fehr, W. R., Caviness, C. E., Burmood, D. T., and Pennington, J. S. 1971. Stage of development descriptions for soybeans, Glycine max (L.) Merrill. Crop Sci. 11:929-931.

7. Johnson, A. B., Scott, H. D., and Riggs, R. D. 
1994. Response of soybean in cyst nematodeinfested soils at three soil-water regimes. J. Nematol. 26:329-335.

8. Kim, K. S., and Riggs, R. D. 1992. Cytopathological reactions of resistant soybean plants to nematode invasion. Pages 157-168 in: Biology and Management of the Soybean Cyst Nematode. R. D. Riggs and J. A. Wrather, eds. American Phytopathological Society Press, St. Paul, MN.

9. Niblack, T. L., ed. 1993. Protect Your Soybean Profits: Manage Soybean Cyst Nematode. American Soybean Association. University of Missouri Press, Columbia.

10. Niblack, T. L., ed. 1999. Soybean Cyst Nematode Management Guide. N. Cent. Soybean Res. Prog. Publ. University of Missouri Print- ing Services, Columbia.

11. Niblack, T. L., Heinz, R. D., Smith, G. S., and Donald, P. A. 1993. Distribution, density, and diversity of Heterodera glycines populations in Missouri. Ann. Appl. Nematol. 25:880-886.

12. Niblack, T. L., Hussey, R. S., and Boerma, H.

R. 1986. Effects of environments, Meloidogyne incognita inoculum levels, and Glycine max genotype on root-knot nematodesoybean interactions in field microplots. J. Nematol. 18:338-346.

13. Seinhorst, J. W. 1965. The relation between nematode density and damage to plants. Nematologica 11:137-154.

14. Smith, G., Wiebold, W., Niblack, T. L., Scharf, P., and Blevins, D. 2000. Yield components of soybean plants infected with soybean cyst nematode and sprayed with foliar applications of boron and magnesium. J. Plant Nutr. 23:827-834.

15. Workneh, F., Tylka, G. L., Yang, X. B., Faghihi, J., and Ferris, J. M.. 1999. Regional assessment of soybean brown stem rot, Phytophthora sojae, and Heterodera glycines using area-frame sampling: Prevalence and effects of tillage. Phytopathology 89:204-211.

16. Young, L. D. 1996. Yield loss in soybean caused by Heterodera glycines. Suppl. J. Nematol. 28:604-607.

17. Young, L. D., and Heatherly, L. G. 1988 Soybean cyst nematode effect on soybean grown at controlled soil water potentials. Crop Sci. 28:543-545. 